\title{
BIBECHANA
}

A Multidisciplinary Journal of Science, Technology and Mathematics

ISSN 2091-0762 (online)

Journal homepage: http://nepjol.info/index.php/BIBECHANA

\section{Photo and electroluminescence of porous silicon layers}

\author{
E. Haji-Ali \\ Department of Physics; Imam Hussein University; Tehran; Iran \\ e-mail: ehajiali@yahoo.com \\ Article history: Received 15 June, 2011; Accepted 11 July, 2011
}

\begin{abstract}
Porous silicon layers were prepared by both chemical and electrochemical methods on $n$ - and $p$ type Si substrates. In the former technique, light emission was obtained from p-type and n-type samples. It was found that intense light illumination during the preparation process was essential for PSi formation on n-type substrates.An efficient electrochemical cell with some useful features was designed for electrochemical etching of silicon. Various preparation parameters were studied and photoluminescence emissions ranging from dark red to light blue were obtained from PSi samples prepared on $\mathrm{p}$-type substrates. $\mathrm{N}$-type samples produced emissions ranging from dark red to orange-yellow. Electroluminescence of porous silicon samples showed that the color of the emission was the same as the photoluminescence color of the sample, and its intensity and duration depended on the current density passed through the sample. The effects of exposure of samples to air, storage in vacuum, and heat-treatment in air on luminescence intensity of the samples and preparation of patterned porous layers were also studied
\end{abstract}

Keywords: Porous silicon layers; photoluminescence; electroluminescence

\section{Introduction}

Silicon is the most important material in microelectronics technology. However, it has a major shortcoming that prevents development of large-scale fabrication of fully-integrated optoelectronic circuits based on silicon. The origin of this drawback is the indirect energy band gap of silicon, which leads to very low quantum efficiencies for photoluminescence (PL) and electroluminescence (EL) processes in silicon. Furthermore, Si has a relatively small $(\sim 1.1 \mathrm{ev})$ band gap. Therefore, the band-to band luminescence of bulk Si occurs in the near-infrared (NIR) part of the spectrum. This makes $\mathrm{Si}$ unsuitable for optical display applications. Consequently, Si was regarded as an unsuitable material for optical applications for many years. On the other hand, GaAs which has a direct band gap and can emit light rather efficiently is expensive and not compatible with the highly developed microelectronics technologies, which are based on silicon. In 1990 Canham [1] reported emission of visible light from highly porous silicon (PSi) samples. Since then the interest on PSi has greatly intensified, and during the last five years considerable research has been done on PSi. These have been aimed at both understanding the phenomenon from the basic science point of view, and also exploring possible optoelectronics applications of this material.

Due to a very large surface area, PSi layers are highly reactive and can be oxidized at low temperatures. Furthermore, $\mathrm{PSi}$ has a higher resistivity than a comparable $\mathrm{SiO}_{2}$ layer. Utilizing 
these properties, "Silicon-On-Insulator" (SOI) and "Fully-Isolated-Porous-Oxided-Silicon" (FIPOS) processes have used PSi for device isolation in Si integrated circuits.

Crystalline $\mathrm{Si}$ can be made porous by partial dissolution in aqueous hydrofluoric (HF) acid. PSi layers have been prepared by both chemical and electrochemical methods. In electrochemical technique, the HF solution etches an array of holes (pores) into the surface of the Si substrate, when an electrical current passes through the cell. The result is an array of pores that are a few nanometers wide and, in some cases, microns long. The Si skeleton that is left behind consists of columnlike structures (a few $\mathrm{nm}$ wide) and many Si microcrystals (with sizes ranging from $\sim 3-$ $20 \mathrm{~nm}$ ) that are not connected with the columnlike structures. Both of these columnlike and particlelike structures have been observed by TEM and SEM studies [2]. The columnlike Si structures that are sometimes referred to as "quantum wires" are highly strained, but retain the crystallinity of the substrate.

Theoretical considerations (based on quantum confinement model) show that when the average width of the Si quantum wires become smaller than about $4 \mathrm{~nm}$, the PSi layer should emit visible light at room temperature [3]. The color of this emission should vary with change in size of the wires, i.e. by making smaller structures; shorter-wavelength $\mathrm{PL}$ emissions should be obtained. These have been confirmed experimentally; Si wire dimensions have been varied with changing PSi formation parameters.

PSi pore diameters are increased with increasing current densities for both $n$ - and p-type samples. At low-current densities the pores are filamentlike and randomly directed. But at higher current densities, the pores "pipe" i.e. becomes larger and more cylindrically shaped [4].

For $p$-type samples both the pore diameters and interpore spacings are extremely small $(\sim 1-5$ $\mathrm{nm}$ ) with a highly interconnected and homogeneous pore network. The pore diameters and also interpore spacings increase slightly with increasing dopant concentration. For n-type Si the pore diameters are considerably larger than $\mathrm{p}$-type $\mathrm{Si}$ and show a strong tendency to form straight channels at low dopant concentration. As the dopant concentration is increased, both the pore diameters and interpore spacings are decreased, to the point where the pore morphologies and diameters of $n+$ and $p+$ samples become comparable [4].

The actual pore formation mechanism shows considerable anisotropy. For example, it is shown that in $\mathrm{n}$-type $\mathrm{Si}$, pore formation results in rectangular-shaped pores [4].

\section{Experimental results}

\subsection{Chemical method}

PSi layers were prepared by etching of $\mathrm{Si}$ in a mixture of $\mathrm{HF}: \mathrm{HNO}_{3}: \mathrm{H}_{2} \mathrm{O}$ (with 1:3:5 ratio) and a small amount of ethanol $(\sim 3 \%)$. Si samples were first cleaned with RCA1 and RCA2 solutions, followed by a dip in a $10 \% \mathrm{HF}$ solution for one minute. Samples were etched for 5 minutes and wear rinsed in deionized water and dried with $\mathrm{N}_{2}$ gas. The etching process was performed under bright light illumination.

Samples emitted organe light under UV illumination. Both $n$ - and $p$ type samples showed light emission, but p-type samples had PL intensities greater than that of the n-type specimens. However these intensities were significantly lower than that of samples prepared by electrochanical technique. This is known to be due to the lower PSi film thicknesses that are attainable by this preparation technique. The film thickness in this process is self limiting and saturates after several minutes; this happens when dissolution rates of $\mathrm{Si}$ at the top surface and at the bottom part of the PSi layer are balanced. 
It is believed that in this case the chemical etching is a two-step process, in which the Si surface is first oxidized by $\mathrm{HNO}_{3}$ acid, and then the oxide is reacted with $\mathrm{HF}$ acid to form $\mathrm{H}_{2} \mathrm{SiF}_{6}$ which is soluble in water. Shih et. al. [5] have proposed a localized electrochemical process for the above PSi formation. According to them, anode and cathode sites are momentarily formed on the etched surface, and a local current flow between them during the etching process. The anodeforming reaction consists of the dissolution of $\mathrm{Si}$, and the cathode reaction is believed to be a complicated reduction of $\mathrm{HNO}_{3}$ that causes holes to be injected in the $\mathrm{Si}$.

The parameters involved are: $\mathrm{HNO}_{3}$ concentration in the enchant mixture, reaction time, stirring condition, and doping level and conductivity type of the substrate. For stronger stirring, a thicker final film is formed; Shi et al [5] have reported PSi formation with p-type samples only. We also found that when the etching process was carried out in dark (or low-level illumination) PSi did not from on n-type samples; However, PSi formation on n-type Si was possible under rather high illumination intensities.

\subsection{Electrochemical method}

Figure (1) shows schematic diagram of the cell that was designed and made for electrochemical etching of Si samples. An ultrasonic vibrator is used for prevention of gas bubble formation on the sample. This cell needs very little (about $15 \mathrm{cc}$ ) etching solution for each sample; And PSi layer can be prepared under illumination (total-surface or patterned), or in the dark.

A Si wafer was first cleaned by RCA 1 and RCA2 solution followed by dipping in a $10 \% \mathrm{HF}$ solution, rinsing in deionized water, and drying by $\mathrm{N}_{2}$ gas. Immediately after cleaning, the wafer was placed in a vacuum evaporation system, and a layer of ohmic contact metal (Al for p-type and Ti/Al for n-type samples) was evaporated on the back side of the wafer. The wafer was then cut into $2 * 2 \mathrm{~cm}$. pieces, and each piece was attached by silver conducting paint to a piece of flatplate copper. This assembly was not only strong enough to be put under pressure in the electrochemical cell, but produced a uniform potential over the whole surface of the wafer under bias. (This was necessary for obtaining a uniform PSi thickness over the whole area of the substrate).

The wafer assembly was then placed in the cell. Upon closing the cell cover, an o-ring was pressed onto the surface of Si substrate and isolated and inner section of the cell, which was then filled with HF solution. The bias was established between a platinum wire (as the cathode) and the copper base plate. The outer section of the cell was partially filled with water, so that ultrasonic vibrations could effectively reach the inner section.

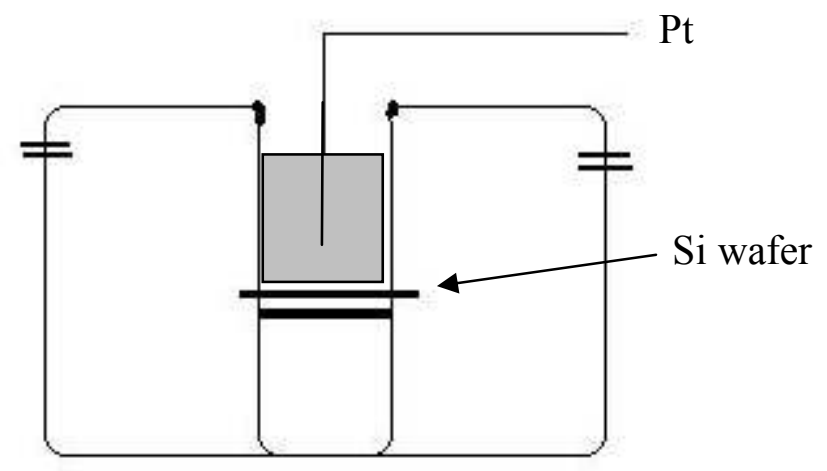

Fig. 1: Schematic diagram of the electrochemical cell used for preparation of PSi layers 
At the end of the anodization process, the sample assembly was removed from the cell, washed and dried (by $\mathrm{N}_{2}$ gas), and finally the $\mathrm{Si}$ substrate was removed from the base plate by using a few drops of acetone to loosen the silver paint.

In this method, the major parameters affecting formation and characteristic of PSi film are: substrate conductivity type, substrate doping level, current density, anodization time, and HF acid concentration, illumination level during the process, and substrate crystal orientation. The effects of these parameters on PSi film have been thoroughly investigated by other workers [6-10]. We also found that PSi film thickness essentially determined by the amount of electric charge passed through the cell (per unit area of the substrate) during formation. This charge is the product of the current density and anodization time. At a fixed HF concentration and current density, PSi film thickness increases linearly with anodization time; and again at the fixed HF concentration, PSi formation rate increases linearly with current density. However, if the anodization current density is increased beyond a certain limit, electropolishing of the $\mathrm{Si}$ surface occurs. There is a transition region between pore formation and electropolishing domains. At a fixed anodization time, the PSi film thickness increases with increasing HF acid concentration in the solution.

The etching mechanism of $\mathrm{Si}$ is a two-step process: First $\mathrm{Si}$ is oxidized by the water content of the HF solution; and then the oxide is etched away by HF acid. It has been suggested that the etching rate is considerably faster than the oxidation rate [7]. Therefore, the amount of the dissolved $\mathrm{Si}$ is determined by the oxidation reaction. It is also suggested that the $\mathrm{Si}$ dissolution during anodization is caused by two reactions: An electrochemical reaction to form PSi (which is proportional to anodization time), and a nonelectrochemical reaction (which dissolves PSi) [7].

Since presence of holes is essential in PSi formation, exposure of the Si substrate to light (with photon energies greater than the $\mathrm{Si}$ bandgap) during anodization process increases hole concentration and therefore, increases the etching rate.

Light exposure during the PSi formation process increased the PL emission intensity and decreased wavelength of the emitted light. Application of an electric field parallel to the surface had a suppressing effect on the PL intensity (which in most cases recovered very slowly when the bias was removed).

\section{3. photoluminescence and electroluminescence results}

\section{1 photoluminescence emission of p-type samples}

PL emissions with colors ranging from dark red to light blue were obtained for $p$ - type samples. In a series of experiments, the total amount of charge passing through the unit area of the samples was kept constant and the current density was varied. The substrates [B-doped, (100), 38-63 $\Omega \mathrm{cm}]$ and the HF concentrations (39\%) were the same for all the tests in each set. Table (1) gives the color of the PL emission of the prepared PSi samples under UV illumination.

Table (1): Color of PL emission of PSi samples (under UV illumination) prepared on p-type Si and under different anodization times. The total charge passed through the cell was constant for all of the cases

\begin{tabular}{|c|c|c|c|}
\hline No & Etching time(minutes) & Current $(\mathrm{mA} / \mathrm{cm} 2)$ & PL emission color \\
\hline $\mathbf{1}$ & 60 & 5 & Dark-red \\
\hline $\mathbf{2}$ & 30 & 10 & Red-orange \\
\hline $\mathbf{3}$ & 15 & 20 & Orange \\
\hline $\mathbf{4}$ & 7.5 & 40 & Orange-yellow \\
\hline $\mathbf{5}$ & 3.75 & 80 & Yellow-green \\
\hline $\mathbf{6}$ & 3.53 & 85 & Light- blue \\
\hline $\mathbf{7}$ & 3 & 100 & --- \\
\hline
\end{tabular}


The samples with yellow to blue PL emissions persisted only for a short duration, and their PL emission could only be seen if the samples were examined immediately after the preparation. The PL of samples with yellow-green emission was gone after about 5 minutes exposure to air; and the blue emission vanished in much shorter times. On the other hand, the PL intensities of samples with orange or red emissions were not destroyed with storage of samples in room air (even for periods of a year or longer). Furthermore, storage of PSi samples with shorter wavelength emissions in vacuum did not diminish their PL emission intensities; and even after several hours, no significant change in the intensities was observed.

The above observations can be attributed to the extremely small thicknesses of $\mathrm{Si}$ wires and microcrystals when the anodization current density is large, i.e. approaching the electropolishing domain. These very thin wires eventually oxidize completely during PL emission intensities; and even after several hours, no significant change in the intensities was observed.

The above observations can be attributed to the extremely small thicknesses of $\mathrm{Si}$ wires and microcrystals when the anodization current density is large, i.e. approaching the electropolishing domain. These very thin wires eventually oxidize completely when the PSi layer is exposed to air. On the other hand, thick $\mathrm{Si}$ wires do not oxidize entirely and a thin $\mathrm{SiO}_{2}$ layer, which forms on the outer surface of the wires, prevents their further oxidation.

Of course, if the temperature is increased, the oxidation process will propagate further until the entire Si structure is oxidized. This was actually observed experimentally; PSi samples with red $\mathrm{PL}$ emissions (which are normally stable) were heated in air. When the temperature reached $\sim 60$ ${ }^{0} \mathrm{C}$ the PL intensity of the sample started to diminish; At $\sim 200{ }^{\circ} \mathrm{C}$ (after 6 minutes total heating time) the emission was completely destroyed. Heating similar samples in boiling water also showed the same oxidation effect.

At current densities of $100 \mathrm{~mA} / \mathrm{cm}^{2}$ or greater (sample No. 7 in Table 1) electropolishing process takes place and PSi is not formed.

The emission intensities for samples 2 and 3 (Table 1) were stronger than the others. The intensities of samples 5 and 6 were lower, though quite visible. Viewing the spectrum of the emitted PL emission of PSi samples by a spectroscope showed that the light consisted of a range of wavelengths, as is well documented in the literature.

\subsection{Photoluminescence emission of $\mathbf{n}$-type samples}

Under various preparation conditions, n-type samples did not produce any visible PL emission, when samples were prepared in the dark. However, PSi was formed and strong PL emissions were observed when the anodization process was done under illumination. This was well observed when only a part of the substrate was illuminated by a bright light source. The contrast between the two parts was quite evident when the sample was inspected under normal or UV illumination.

Again, by varying anodization current densities (under a constant-charge condition), various PL colors were obtained from these samples. Table (2) shows the results. The samples were illuminated by a $150 \mathrm{~W}$ mercury-vapor lamp at $30 \mathrm{~cm}$. distance the PSi formation. 


\section{E. Haji-Ali / BIBECHANA 8 (2012) 46-52 : BMHSS, p.51}

Table (2): Color of PL emission of PSi samples (under UV illumination) prepared on n-type Si and under different anodization times. The total charge passed through the cell was constant for all of the cases

\begin{tabular}{|c|c|c|c|}
\hline No & Etching time(minutes) & Current $(\mathrm{mA} / \mathrm{cm} 2)$ & PL emission color \\
\hline 1 & 50 & 4 & Dark-red \\
\hline 2 & 34 & 6 & Red-orange \\
\hline 3 & 25 & 8 & Orange-yellow \\
\hline
\end{tabular}

Pattered PSi layers were generated by two techniques. First, a layer of positive photoresist was patterned by photolithographic method on the substrate surface.

In another technique, patternd illumination of the sample (during the anodization process) was used. A mask was placed in the path of light (see Fig. 2), and its image was optically projected on the Si substrate. However, due to scattering of light in the solution and body of the cell, pattern definition was not as good as the former technique.

\section{3 electroluminescence results}

Three kind of EL cells (electrolyte/PSi, Au/PSi, and ITO/PSi) were made and studied. The electrolyte for the first type consisted of acid solutions, usually HNO3: $\mathrm{H} 2 \mathrm{O}$ (1:5 ratios). The samples were placed in the same cell that was used for preparation of PSi samples (see Fig. 1). The bias was established between the top Pt electrode and the base of the sample, i.e. ohmic contact with Si substrate.

The amount of gold that was thermally evaporated on the PSi layer in vacuum was adjusted so that the Au electrode acted as a semi-transparent electrode in Au/PSi cells. The third kind of EL cells were prepared by deposition of an indium oxide (IO) or indium-tin-oxide (ITO) layer on top of the PSi samples. Since during the deposition of IO or ITO layers (by reactive evaporation of In metal in partial pressure of oxygen and spray-hydrolysis methods, respectively) the substrate needed to be heated to several hundred degrees Celsius, the PSi layer oxidized during the transparent electrode deposition, and therefore, no visible EL emission was observed from these cells.

Of the other two types of EL cells, only electrolyte/PSi cells produced bright visible EL emissions. The intensity of the emission increased with increasing current passing through the cells. The color of the emission was the same as the PL color of the sample. However, due to electrochemical reaction between the electrolyte and PSi layer (which dissolves PSi), the lifetime of these cells was limited to a few minutes; after which, the EL emission sharply reduced to zero.

\section{Conclusion}

Porous silicon is fast becoming an important research subject. This is not only due to its potential applications in optoelectronic devices and integrated optics circuits, but also because of its significance from the basic solid state science point of view.

In this article, we report: (1) By shining intense light on the sample during the PSi preparation process, it is possible to form PSi layer on n-type silicon substrates as well; (2) Features of an efficient electrochemical cell for preparation of PSi samples, which is simple to make, but has various useful capabilities; (3) Obtaining PL and EL emissions ranging from dark red to light blue from PSi samples is possible by just changing the preparation parameters during PSi formation process (with no need for post-deposition heat-treatment of samples in boiling water). Furthermore, preparation and characterization results for various PSi samples are discussed. 


\section{Acknowledgement}

The author would like to thank Dr. Mahdi Eshghi for the positive suggestions which helped to improve the present work.

\section{References}

[1] L. T. Canham, Appl. Phys. Lett. 57(10), (1990) 1046.

[2] X. Wang, Modern Phys. Lett. B 8 (2), 69 (1994) 1597

[3] L. T. Canham, Phys. World, 41(March 1992) 1540.

[4] R. L. Smith and S. D. Collins, J. Appl. Phys. 71 (8), R1 (1992).

[5] S. Shih, et al., Mat. Res. Soc. Symp. Proc. 256, 27 (1992) 197.

[6] T. Asano, et al., Jpn. J. Appl. Phys. 31, L373 (1992) 865.

[7] H. Unno, K. Imai, \& S. Muramoto, J. Electrochem. Soc. S. S. \& T 134 (3), 645(1987) 645 648.

[8] J. T. Lue, et al., Solid State Commun. 86(9), (1993) 593.

[9] S. F. Chung, S. D. Collins, \& R. L. Smith, Appl. Phys. Lett. 55(7), (1989) 675.

[10] O. Teschke, M. C. Goncalves, \& F. Galembeck, Appl. Phys. Lett. 63(10), (1993) 1348. 